\title{
MUCOCELE ESFENOIDAL EN NIÑA DE SIETE AÑOS
}

Jimmy de J esús M encías M D*, Ricardo Enrique Guerra M D**, Perla Villamor M D***, Carolina Rojas M D ***

\section{Resumen}

Los mucoceles del seno esfenoidal representan de 1 a $2 \%$ de todos los de los senos paranasales. Se han reportado 140 casos de los cuales $95 \%$ ocurrieron en adultos, siendo un paciente de 11 años el más joven informado. El objetivo es presentar nuestra experiencia en una paciente de siete años de edad con un mucocele del seno esfenoidal. Los signos y síntomas consistieron en cefalea y rinorrea purulenta escasa. El diagnóstico se realizó mediante TAC y RMN de senos paranasales, así como por los hallazgos histopatológicos. El tratamiento consistió en abordaje endoscópico. El diagnóstico precoz es importante para evitar déficit neurológico permanente o alteraciones visuales y nerviosas por vecindad. Se analizó la etiología, manifestaciones y tratamiento de los mucoceles esfenoidales y se revisa la literatura médica disponible.

Palabras clave: mucocele, seno esfenoidal, esfenoides, niños, pediátrico, infancia.

\section{A CASE OF SPHENOID MUCOCELE IN A SEVEN-YEAR-OLD GIRL}

\section{Abstract}

Sphenoid sinus mucoceles represent only 1 to $2 \%$ of all paranasal mucoceles. Only 140 cases have been reported so far, $\mathbf{9 5 \%}$ in adults and an 11-year-old child is the youngest patient reported. Our objective is to describe our experience in a 7-year-old girl with a sphenoid sinus mucocele. Signs and symptoms consisted of headache and mild purulent rhinorrhea. Paranasal sinus CT and MR imaging, as well, as the histopathologic findings allowed the diagnosis. Patient was treated by endoscopic approach. Because of close proximity with vital structures early diagnosis is essential to prevent permanent neurologic deficit, such as, visual or nerve impairment. Etiology, manifestations and treatment of sphenoid sinus mucoceles and relevant medical literature were reviewed.

Key words: mucocele, sphenoid sinus, sphenoid, children, pediatric, childhood.

Fecha recibido: agosto I de 2014 - Fecha aceptado: marzo 12 de 2015

* Médico Otorrinolaringólogo, Hospital Infantil Universitario de San José. Instructor Asistente, Fundación Universitaria de Ciencias de la Salud. Bogotá DC, Colombia.
** Residente II de Otorrinolaringología, Fundación Universitaria de Ciencias de la Salud. Bogotá DC, Colombia.

*** Residente III de Otorrinolaringología, Fundación Universitaria de Ciencias de la Salud. Bogotá DC, Colombia. 


\section{Introducción}

L os mucoceles son masas encapsuladas, revestidas por un epitelio no neoplásico, expansibles y localmente destructivas, que contienen casi siempre moco estéril. Solo de 1 a $2 \%$ de todos los mucoceles de los senos paranasales se encuentran en el esfenoidal y hasta la fecha 140 casos han sido reportados, 95\% en pacientes adultos. ${ }^{1,2}$

EI origen sigue siendo controvertido, parece estar relacionado con un proceso inflamatorio crónico que ocurre en un espacio cerrado, causando obstrucción del ostium que conduce a la acumulación de secreciones mucosas en el seno paranasal. Puede cursar con un tumor benigno, cicatrices postraumáticas 0 inflamación crónica en el 61 a 80\% de los casos. ${ }^{3,4}$ L a característica histopatológica más evidente es la presencia de un epitelio columnar pseudoestratificado ciliado. ${ }^{5}$

Por lo regular los mucoceles son asintomáticos y se identifican en forma casual después de la realización de una TAC o una RM N de cráneo o senos paranasales. Sin embargo, cuando hay síntomas, suelen ser inespecíficos como resultado de la presión mecánica sobre las estructuras vecinas o por compromiso de los nervios en el proceso inflamatorio. Los más co- munes son cefalea, pérdida de la visión y parálisis de III y VI pares craneales. ${ }^{6-8} \mathrm{EI}$ tratamiento de elección es la esfenoidectomia endoscópica realizando marsupialización de la lesión con reti ro completo de su capsula, aunque se reporta un alto porcentaje de recaídas postratamiento. ${ }^{9}$ El diagnóstico diferencial incluye sinusitis crónica esfenoidal, bolas fúngicas, neoplasias benignas como el papiloma invertido y rara vez tumores malignos. ${ }^{10}$

\section{Presentación del caso}

Paciente de siete años de edad, estudiante, natural y residente de B oyacá (Colombia) asiste al servicio de urgencias del Hospital Infantil U niversitario de San J osé en Bogotá DC, Colombia, en compañía de su madre con un cuadro clínico de 14 días de evolución caracterizado por dolor retrocular y cefalea frontal intensa progresiva, con intensidad al ingreso de 8/10, persistente, asociada con rinorrea purulenta escasa. A I interrogatorio no se indicó la presencia de obstrucción nasal, emesis, epistaxis o cambios visuales. A ntecedentes médi cos negativos. A I examen físico se encontró una paciente alerta, orientada, afebril, sin signos de dificultad respiratoria, rinoscopia anterior con septum integro, central, cornetes edematosos, rinorrea purulenta escasa, sin epistaxis. Se solicitó TAC cerebral simple evidenciándose lesión expansiva
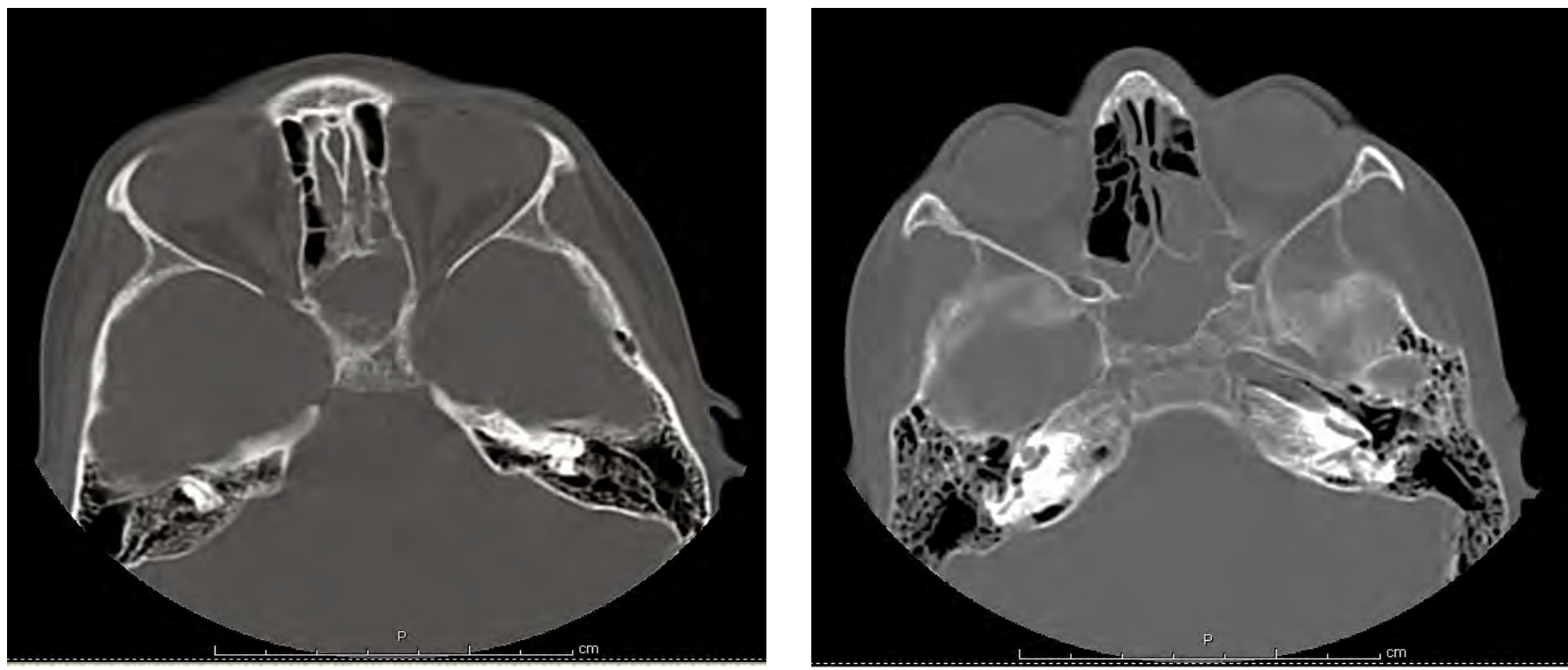

Figura 1. TAC simple de cerebro: lesión expansiva con densidad de tejidos blandos que ocupa el seno esfenoidal en su totalidad. 
con densidad de tejidos blandos que ocupaba el seno esfenoidal en su totalidad (Figura 1). Se realizaron estudios complementarios como nasosinuscopia visualizando como único hallazgo positivo rinorrea blanquecina proveniente de cel dillas etmoidales posteriores. L a R M N de senos paranasales con contraste evidencia expansión de las paredes del seno esfenoidal y ocupación difusa del mismo por material de aspecto homogéneo compatible con mucocele (27 $\mathrm{mm}$ en eje craneocaudal, $26 \mathrm{~mm}$ en el transverso y $20 \mathrm{~mm}$ anteroposterior) (Figura 2). Por lo anterior y ante la sintomatología persistente se decidió realizar esfenoidectomia y etmoidectomia anterior y posterior para drenaje y permeabilización del seno esfenoidal. En la cirugía se observó una lesión expansiva mucoide con revestimiento capsular epitelial y reabsorción ósea asociada. Se realizó drenaje y limpieza de seno esfenoidal con permeabilización del ostium (Figura 3). La evolución fue satisfactoria con resolución completa de la sintomatología. La estancia intrahospitalaria fue de cuatro días.
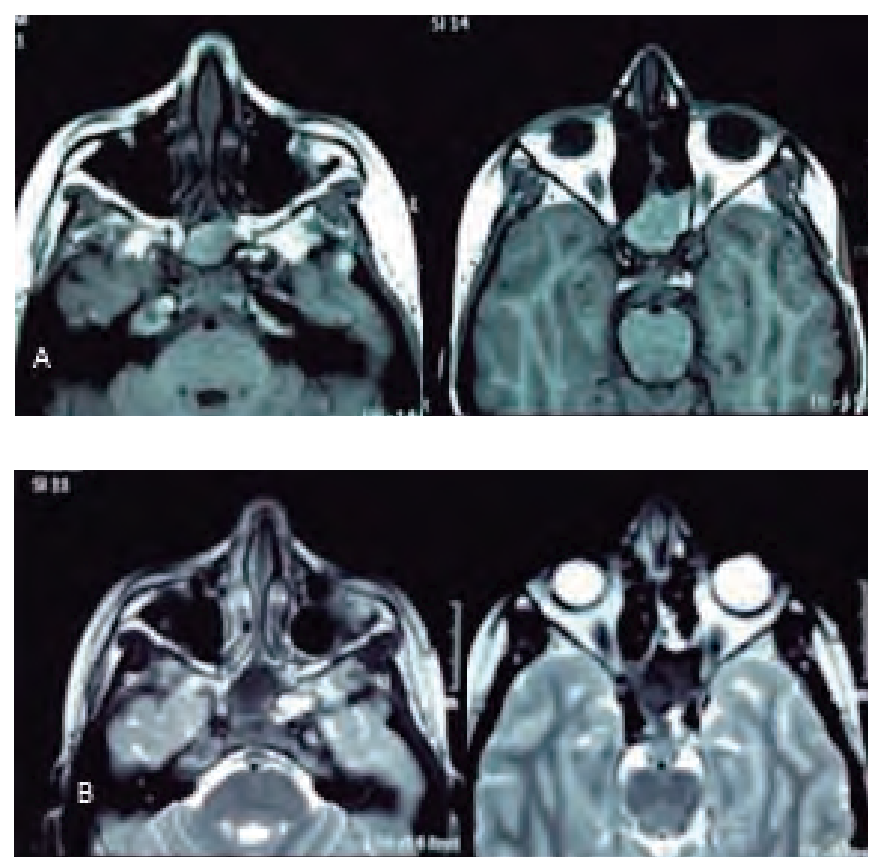

Figura 2. RMN de senos paranasales con contraste. Expansión de las paredes del seno esfenoidal y ocupacion difusa del mismo por material de aspecto homogéneo compatible con mucocele esfenoidal, $27 \mathrm{~mm}$ en eje craneocaudal, $26 \mathrm{~mm}$ en el transverso y $20 \mathrm{~mm}$ el anteroposterior. Lesión isointensa en TI (A), e hipointensa en T2 (B) por el alto contenido de proteina en el moco.

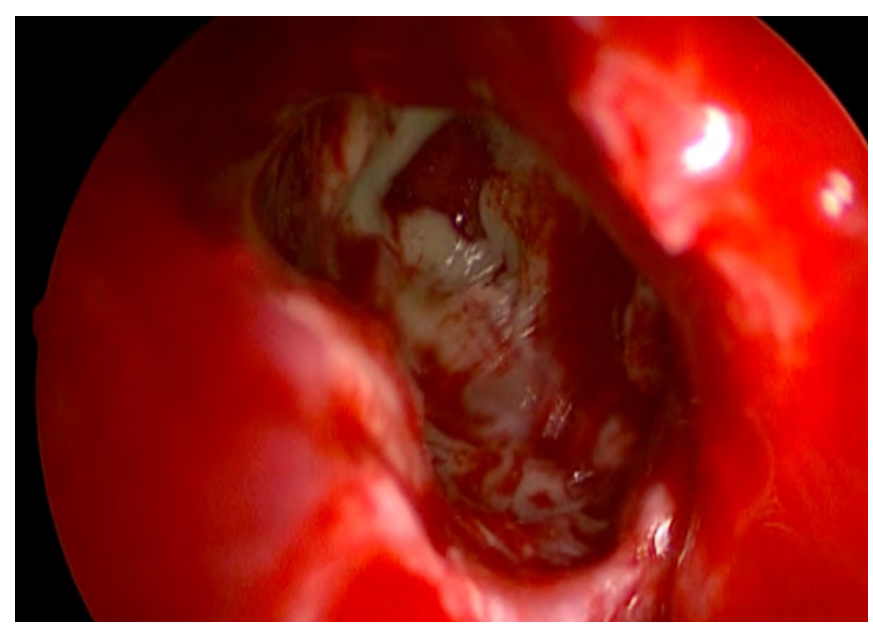

Figura 3. Seno esfenoidal después de la resección del mucocele y permeabilización del ostium. Se evidencia osteítis y cambios erosivos óseos locales.

\section{Discusión y conclusiones}

EI mucocele del seno esfenoidal es una entidad poco frecuente, la mayoría ocurre en adultos y puede dar lugar a secuelas graves si el diagnóstico y el tratamiento se retrasan. En la población pediátrica es rara, sin embargo, merece especial atención debido a su ubicación en la base del cráneo. ${ }^{11,12}$ L a etiología es todavía desconocida, pero se han descrito diferentes factores infecciosos o traumáticos como posibles causantes, con inflamación crónica en 61 a 80\% de los casos, produciendo obstrucción del ostium que conduce a la acumulación de secreciones mucosas., $\mathrm{L}$ a tasa de crecimiento lento y su tamaño variable son responsables del amplio espectro de síntomas y características clínicas. ${ }^{4}$ Las más frecuentes son cefalea localizada, dolor retrocular, sinusitis persistente que no responde a la terapia médica con secuelas neurológicas en algunos casos como atrofia del nervio óptico, parálisis de pares craneales (en especial III y VI), meningitis, abscesos intracraneales, neumoencéfalo y hemiparesia.

La TAC muestra una masa de tejidos blandos isointensa con un adelgazamiento de los márgenes óseos. El aspecto en la RM N es variable, dependiendo de la concentración de proteína y la viscosidad de los contenidos de la lesión. Por lo general son de al ta intensidad en T2 y sus características pueden variar en T1.1,11 La mayor controversia en cuanto al manejo del mucocele 
esfenoidal es la indicación quirúrgica. La literatura internacional indica que los pacientes deben ser tratados mediante abordaje quirúrgico endoscópico, excepto cuando el compromiso intracraneal o intraorbitario es extenso.

La marsupialización endoscópica transnasal ofrece una solución mínimamente invasiva simple y eficaz. L os sitios de displasia deben ser tratados solo en caso de impacto estético o funcional. La vigilancia debe ser prolongada en estos pacientes, cuya evolución es impredecible. ${ }^{12}$ Como se ilustra con este caso, los mucoceles del seno esfenoidal pueden presentarse en población pediátrica con sintomatología principal de cefal ea intensa localizada. La TAC y la R M N proveen datos relevantes para su diagnóstico y planeamiento quirúrgico.

\section{Referencias}

1. Kösling S, Hintner M, Brandt S, Schulz T, Bloching M. M ucoceles of the sphenoid sinus. Eur J Radiol. 2004 Jul;51(1):1-5.

2. Soon SR, Lim CM, Singh H, Sethi DS. Sphenoid sinus mucocele: 10 cases and literature review. J Laryngol Otol. 2010 Jan;124(1):44-7.

3. Devars du Mayne $M$, M oya-Plana A, M alinvaud D, Laccourreye 0 , B onfils $P$. Sinus mucocele: natural history and long-term recurrence rate. Eur A nn Otorhinolaryngol Head Neck Dis. 2012 J un;129(3):125-30.

4. Giovannetti F, Filiaci F, Ramieri V, Ungari C. Isolated sphenoid sinus mucocele: etiology and management. J Craniofac Surg. 2008 Sep;19(5):1381.

5. Lund VJ. Fronto-ethmoidal mucoceles: a histopathological sinuses. J Laryngol Otol 1991;105:921-923.

6. Kataria R, Gupta S, Chopra S, Bagaria H, Sinha VD. M ucocele of the sphenoid sinus: A rare cause of reversible 3(rd) nerve palsy. A nn Indian A cad N eurol. 2012 A pr;15(2):158-60.

7. Cheng CS, Sanjay S, Y ip CC, Yuen HW. Sphenoidal mucocele presenting as acute cranial nerve palsies. Saudi J Ophthalmol. 2012 0ct;26(4):459-61.

8. Sharifi G, Jalessi M, Erfanian D, Farhadi M. Sudden blindness due to isolated sphenoid sinus mucocele and retention cyst. B raz J Otorhinolaryngol. 2013. JanFeb;79(1):120.

9. Facon F, Nicollas R, Paris J, y col. Surgery of mucocele of the paranasal sinuses: report of 52 cases with a middle term follow up. Rev L aryngol Otol R hinol (Bord) 2008;129:167-73.

10. Grillone GA, Kasznica P. Isolated sphenoid sinus disease. Otol Clin N A m 2004;37:435-451.

11. Lui YW, Dasari SB, Young RJ. Sphenoid masses in children: radiologic differential diagnosis with pathologic correlation. AJNR A m J Neuroradiol. 2011 A pr;32(4):617-26.

12. Zaki Z, B elcaid A, A lami M N. A rare cause of craniofacial pain in children. Eur A nn Otorhinolaryngol Head Neck Dis. 2013 Feb;130(1):36-38. 